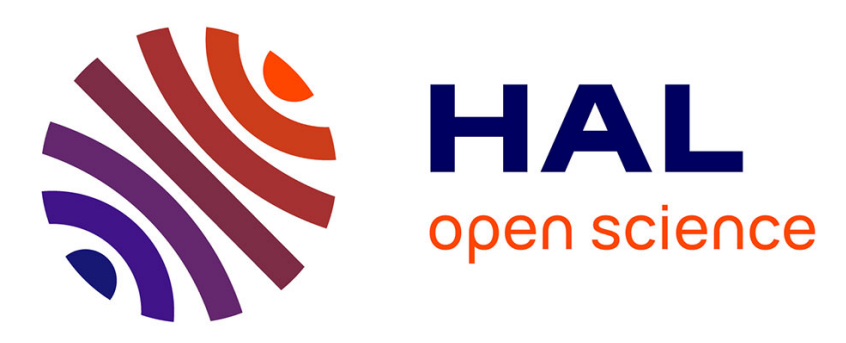

\title{
Une méthode de calcul de structures soumises a des chargements mobiles
}

\author{
Mac-Lan Nguyen-Tajan, Habibou Maitournam, Jean Jacques Thomas
}

\section{To cite this version:}

Mac-Lan Nguyen-Tajan, Habibou Maitournam, Jean Jacques Thomas. Une méthode de calcul de structures soumises a des chargements mobiles: Application au freinage automobile. Revue Européenne des Éléments Finis, 2002, 11, pp.247-261. 10.3166/reef.11.247-261 . hal-00111353

\section{HAL Id: hal-00111353 \\ https://hal.science/hal-00111353}

Submitted on 29 Jul 2019

HAL is a multi-disciplinary open access archive for the deposit and dissemination of scientific research documents, whether they are published or not. The documents may come from teaching and research institutions in France or abroad, or from public or private research centers.
L'archive ouverte pluridisciplinaire HAL, est destinée au dépôt et à la diffusion de documents scientifiques de niveau recherche, publiés ou non, émanant des établissements d'enseignement et de recherche français ou étrangers, des laboratoires publics ou privés. 


\title{
Une méthode de calcul de structures soumises à des chargements mobiles
}

\section{Application au freinage automobile}

\author{
Mac-Lan Nguyen-Tajan ${ }^{*, * *}$, Habibou Maitournam**, \\ Jean-Jacques Thomas* \\ * PSA Peugeot Citroën - DINQ/DRIA/SARA/MATE \\ Route de Gisy - 78943 VELIZY-VILLACOUBLAY \\ maclan.nguyen@mpsa.com \\ ** Laboratoire de Mécanique des Solides \\ Ecole Polytechnique \\ 91128 Palaiseau Cedex \\ maclan@Ims.polytechnique.fr
}

RÉSUMÉ. Le travail présenté dans cet article s'inscrit dans une étude globale de la tenue en service d'un disque de frein automobile. Celui-ci subit des chargements thermomécaniques responsables de divers types d'endommagement : faïençage, fissures radiales sur les pistes de frottement, rupture du bol. La prédiction numérique de ces avaries exige d'abord la mise au point de méthodes numériques adaptées aux problèmes de structures soumises à des chargements thermomécaniques mobiles. Celles-ci consistent en des algorithmes eulériens qui sont présentés et décrits.

ABSTRACT. The work presented in this paper is part of a global study of the life prediction of an automotive brake disc. This component is subjected to thermomechanical loads that can generate different types of damage : honey-comb cracking, radial cracks on the rubbing surface or circonferential crack in the elbow. The numerical prediction of these phenomena needs the elaboration of numerical methods adapted to problems of structures submitted to thermomechanical moving loads. These methods consist in eulerian algorithms that are presented and described.

MOTS-CLÉS : algorithmes eulériens, chargement mobile, thermomécanique, disque de frein.

KEYWORDS: eulerian algorithms, moving load, thermomechanics, brake disc. 


\section{Introduction}

Le disque de frein fait partie d'un ensemble de problèmes thermomécaniques plus larges : celui des structures soumises à des chargements mobiles. De manière générale, ces problèmes peuvent faire intervenir des sollicitations mécaniques (roulements à billes, galetage, pneu, rail soumis au roulement et au frottement de la roue...), thermiques (soudage...) ou bien thermomécaniques (disque de frein soumis à la pression des plaquettes et à l'échauffement induit...). Sous l'effet de ces chargements, les structures peuvent plastifier, s'échauffer et subir des phénomènes d'endommagement et de fatigue. Une première étape de leur étude consiste donc en l'évaluation de leurs "états thermomécaniques". Les essais expérimentaux sont souvent coûteux, longs et nécessitent l'utilisation de pièces qui ne sont parfois que des prototypes. L'objectif est alors de mettre en œuvre des simulations numériques pouvant compléter ces essais. Elles doivent fournir une évaluation fiable des champs couplés de température, de contraintes et de déformations plastiques des structures étudiées, en des temps de calculs compatibles avec les exigences des bureaux d'étude.

Les difficultés majeures de la simulation du disque de frein sont les suivantes :

- les modèles Eléments Finis doivent être tridimensionnels. En effet, si le disque de frein plein peut être assimilé à une structure axisymétrique, les chargements, les conditions aux limites et les champs thermomécaniques ne le sont pas ;

- les gradients thermiques et mécaniques engendrés par les frottements et les déformations du disque sont très élevés; le maillage doit être raffiné dans les zones concernées ;

- les couplages sont nombreux. Le contact disque/plaquettes génère de la chaleur mais varie avec les dilatations qu'il induit. Les coefficients thermomécaniques dépendent de la température et étant données les valeurs atteintes, le comportement du matériau n'est plus élastique mais il devient plastique, voire élastoviscoplastique ;

- le problème est instationnaire;

- le chargement est mobile par rapport à la structure et sa vitesse décroît.

Pour modéliser fidèlement le système, la méthode de résolution numérique "classique" consisterait à effectuer un calcul incrémental où le chargement serait translaté à chaque pas de temps. Cette méthode est lente et ne peut pas être envisagée dès lors que l'on veut calculer des modèles à grand nombre de degrés de liberté avec des nonlinéarités telles que la plasticité, le contact ou le couplage thermomécanique.

D'autres méthodes numériques ont été proposées mais elles ne conviennent pas au problème du disque. En effet, l'analyse simplifiée développée par J. Zarka [ZAR 80] permet d'obtenir directement l'état limite d'une structure soumise à des chargements périodiques mais ne donne pas l'évaluation cycle par cycle de sa réponse. Les méthodes ALE (Arbitrory Lagrangian Eulerian) [ASK 97] permettent quant à elles d'aborder les problèmes transitoires à grandes déformations (par exemple, les problèmes de laminage ou d'extrusion). Une méthode d'adaptation de maillage est utilisée afin que les éléments du maillage ne soient pas trop distordus par les grandes déformations 
induites par le chargement. Ce procédé est très coûteux en temps de calcul et souvent, les problèmes sont considérés commes stationnaires. Ces méthodes ALE paraissent trop sophistiquées pour le problème du frein.

En effet, notre but est de développer des algorithmes adaptés aux problèmes de structures subissant des grands déplacements mais des petites transformations, dus à des chargements transitoires. La méthode proposée consiste en un calcul Transitoire dans le Repère du Chargement en Coordonnées Eulériennes (que l'on note brièvement TRC). Cet algorithme permet de prendre en compte l'évolution au cours du temps de l'amplitude du chargement (évolution des contacts, variation du flux thermique...) et celle de sa vitesse de déplacement (dans le cas du freinage, elle est évidemment décroissante!) tout en limitant les temps de calcul. L'idée est de travailler dans le repère du chargement en utilisant les coordonnées eulériennes pour prendre en compte le flux de matière par rapport à ce repère, et de prendre comme configuration de référence celle qui suit le mouvement rigide de la structure. Ainsi, deux premiers avantages peuvent être cités :

- avec ce choix de configuration de référence et avec l'hypothèse des petites transformations, les distorsions des éléments finis sont assez petites pour que le maillage n'ait pas besoin d'être réactualisé régulièrement; il reste fixe et les lourdeurs numériques rencontrées avec les méthodes d'adaptation de maillage sont évitées ;

- si les zones à forts gradients thermomécaniques sont fixes dans ce repère de calcul, le maillage peut n'être raffiné que dans les régions concernées; dans les autres zones, il peut être grossier. C'est un gain en termes d'espace mémoire et de temps de calcul.

La méthode TRC est finalement une généralisation de l'algorithme stationnaire passage par passage proposé par Q.S. Nguyen [NGU 81], K. Dang Van et H. Maitournam [DAN 93] aux problèmes de chargement variable au cours d'un cycle. En effet, la méthode stationnaire permet de résoudre les problèmes de structure soumise à un chargement mobile supposé constant en vitesse et en amplitude au cours d'un passage (il peut par contre varier d'un passage à l'autre). Cet algorithme fournit, en un seul calcul, l'état thermomécanique de la structure après chaque passage du chargement. Il a l'avantage d'être extrêmement rapide par rapport à un calcul incrémental classique et a été appliqué avec succès à de nombreux problèmes industriels.

Dans cet article, nous formulerons dans un premier temps les équations du problème thermoélastoplastique dans le repère du chargement, en petites transformations. Les formulations discrétisées ainsi que les schémas de résolution sont données respectivement pour les algorithmes TRC et stationnaire dans les parties 3 et 4. L'étude comparative entre les algorithmes TRC, stationnaire et "incrémental classique" (basé sur des translations successives du chargement), reposant sur un calcul élastoplastique d'une structure bidimensionnelle rectangulaire, permet de mettre en évidence les différents intérêts des algorithmes. Enfin, l'exemple d'une simulation thermomécanique tridimensionnelle d'un disque de frein plein automobile est brièvement présenté dans la partie 6 . 


\section{Equations du problème}

\subsection{Description du problème}

On considère un milieu continu occupant un domaine $\mathcal{D}$, soumis à un chargement thermique et mécanique mobile. Il s'agit d'écrire les équations du problème dans un repère lié au chargement, en prenant comme configuration de référence la configuration initiale animée du mouvement rigidifiant de la structure dans ce repère.

On note $\mathcal{R}=\left(O, \mathbf{e}_{\mathbf{X}}, \mathbf{e}_{\mathbf{Y}}, \mathbf{e}_{\mathbf{Z}}\right)$ un repère lié au solide et $\mathcal{R}^{\prime}=\left(O^{\prime}, \mathbf{e}_{\mathbf{x}}, \mathbf{e}_{\mathbf{y}}, \mathbf{e}_{\mathbf{z}}\right)$ un repère lié au chargement qui se déplace à la vitesse $\mathbf{V}(t)$ par rapport à $\mathcal{R}$. Dans le repère de calcul $\mathcal{R}^{\prime}$, la structure se déplace donc à la vitesse $-\mathbf{V}(t)$ (Figure 1). Ainsi, la dérivée particulaire de toute grandeur tensorielle $f$ liée à la matière s'écrit sous la forme :

$$
\dot{\mathbf{f}}(\mathbf{x}, t)=\frac{\partial \mathbf{f}}{\partial t}(\mathbf{x}, t)+\nabla_{x} \mathbf{f}(\mathbf{x}, t) \cdot \mathbf{v}(\mathbf{x}, t)
$$

avec :

$$
\mathbf{v}(\mathbf{x}, t)=\mathbf{v}_{r}(\mathbf{x}, t)-\mathbf{V}(\mathbf{x}, t)
$$

où $\mathbf{v}$ désigne la vitesse du point matériel par rapport à $\mathcal{R}^{\prime}$ et $\mathbf{v}_{r}$ sa vitesse par rapport à $\mathcal{R}$.

L'hypothèse des petites transformations dans le repère lié au milieu $\mathcal{D}$ permet de négliger $\mathbf{v}_{\mathbf{r}}(\mathbf{x}, t)$ par rapport à $\mathbf{V}(\mathbf{x}, t)$. Ainsi, l'expression de $\dot{\mathbf{f}}$ se simplifie en :

$$
\dot{\mathbf{f}}(\mathbf{x}, t)=\frac{\partial \mathbf{f}}{\partial t}(\mathbf{x}, t)-\nabla_{\mathbf{x}} \mathbf{f}(\mathbf{x}, t) \cdot \mathbf{V}(\mathbf{x}, t)
$$

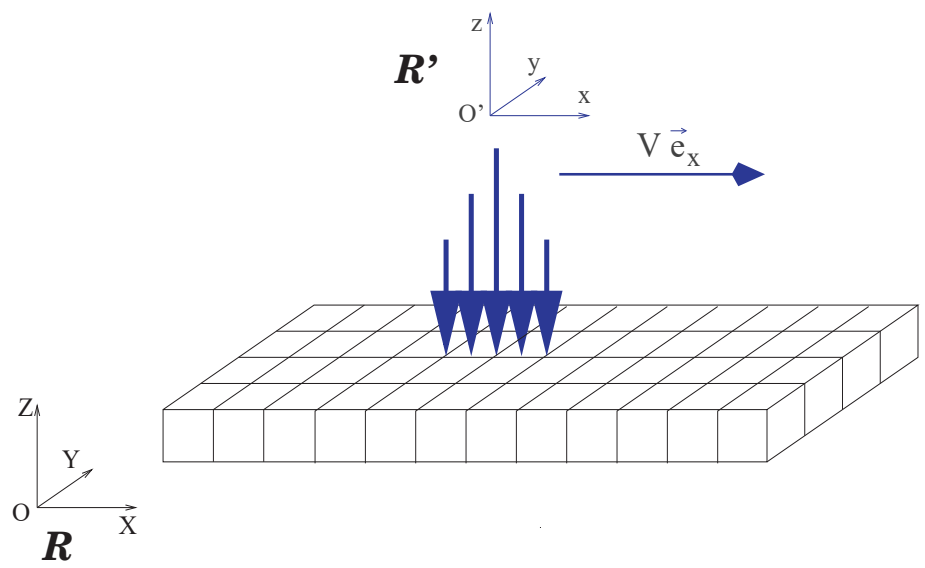

Figure 1. Chargement mobile sur une plaque 


\subsection{Equations du problème thermoélastoplastique}

On se place dans le cadre d'une évolution quasistatique en petites transformations. Le matériau est homogène et isotrope; il obéit à une loi de comportement élastoplastique à écrouissage cinématique linéaire et isotrope de type von Mises et à la loi de conduction de Fourier. Le couplage thermomécanique réside dans la prise en compte des contraintes thermiques et de la dissipation de chaleur générée par le frottement et les déformations plastiques et les variables internes. La production de chaleur par déformations élastiques est négligée. Pour établir les équations d'évolution thermomécanique dans le repère du chargement, il faut expliciter toutes les dérivées temporelles selon l'expression (2). Les équations qui font intervenir les vitesses sont : l'équation d'équilibre (avec le terme d'accélération), la loi d'écoulement plastique et l'équation de chaleur. Avec les notations classiques, on obtient alors :

- Equation d'équilibre

$$
\begin{gathered}
\operatorname{div} \boldsymbol{\sigma}(\mathbf{x}, t)+\mathbf{f}(\mathbf{x}, t)=\rho\left[\frac{\partial^{2} \mathbf{x}}{\partial t^{2}}-2 \nabla\left(\frac{\partial \mathbf{x}}{\partial t}\right) \cdot \mathbf{V}(\mathbf{x}, t)\right. \\
\left.-\nabla \mathbf{x} \cdot \frac{\partial \mathbf{V}}{\partial t}(\mathbf{x}, t)+\nabla(\nabla \mathbf{x} \cdot \mathbf{V}(\mathbf{x}, t)) \cdot \mathbf{V}(\mathbf{x}, t)\right]
\end{gathered}
$$

dans $\mathcal{D}$

- Loi de comportement élastoplastique

$$
\begin{array}{rlr}
\boldsymbol{\sigma}(\mathbf{x}, t) & =\boldsymbol{\sigma}_{o}(\mathbf{x}) & \\
\quad+\mathbf{L}:\left[\boldsymbol{\epsilon}(\mathbf{x}, t)-\boldsymbol{\epsilon}^{p}(\mathbf{x}, t)-\alpha\left(T(\mathbf{x}, t)-T_{o}(\mathbf{x})\right) \mathbf{I}\right] & \text { dans } \mathcal{D} \\
\boldsymbol{\epsilon}=\boldsymbol{\epsilon}^{e}+\boldsymbol{\epsilon}^{p} & \text { dans } \mathcal{D}
\end{array}
$$

- Loi d'écoulement plastique

$$
\begin{array}{ll}
\frac{\partial \boldsymbol{\epsilon}^{p}}{\partial t}(\mathbf{x}, t)-\nabla \boldsymbol{\epsilon}^{p}(\mathbf{x}, t) \cdot \mathbf{V}(\mathbf{x}, t)=\lambda \frac{\partial f}{\partial \boldsymbol{\sigma}}(\mathbf{x}, t) & \text { dans } \mathcal{D} \\
\quad \text { avec } \lambda f=0 ; \lambda \geq 0 ; f \leq 0 & \\
\frac{\partial \alpha_{k}}{\partial t}(\mathbf{x}, t)-\nabla \alpha_{k}(\mathbf{x}, t) \cdot \mathbf{V}(\mathbf{x}, t)=-\lambda \frac{\partial f}{\partial A_{k}}(\mathbf{x}, t) & \text { dans } \mathcal{D}
\end{array}
$$

- Compatibilité

$$
\boldsymbol{\epsilon}(\mathbf{x}, t)=\frac{1}{2}\left[\nabla \mathbf{u}(\mathbf{x}, t)+{ }^{t} \nabla \mathbf{u}(\mathbf{x}, t)\right]
$$

dans $\mathcal{D}$

- Conditions aux limites mécaniques

$$
\begin{cases}\boldsymbol{\sigma}(\mathbf{x}, t) \cdot \mathbf{n}(\mathbf{x}, t)=\mathbf{F}^{\mathbf{d}}(\mathbf{x}, t) & \text { sur } \partial \mathcal{D}_{F} \\ \mathbf{u}(\mathbf{x}, t)=\mathbf{u}^{\mathbf{d}}(\mathbf{x}, t) & \text { sur } \partial \mathcal{D}_{u}\end{cases}
$$

- Equation thermique

$$
\begin{aligned}
k \Delta T(\mathbf{x}, t)- & \rho c \frac{\partial T}{\partial t}(\mathbf{x}, t)+\rho c \nabla T(\mathbf{x}, t) \cdot \mathbf{V}(\mathbf{x}, t) \\
=-r(\mathbf{x}, t)- & \boldsymbol{\sigma}(\mathbf{x}, t):\left(\frac{\partial \boldsymbol{\epsilon}^{p}}{\partial t}(\mathbf{x}, t)-\nabla \boldsymbol{\epsilon}^{p}(\mathbf{x}, t) \cdot \mathbf{V}(\mathbf{x}, t)\right) \\
& +A_{k}(\mathbf{x}, t):\left(\frac{\partial \alpha_{k}}{\partial t}(\mathbf{x}, t)-\nabla \alpha(\mathbf{x}, t) \cdot \mathbf{V}(\mathbf{x}, t)\right) \quad \text { dans } \mathcal{D}
\end{aligned}
$$

- Loi de Fourier

$$
\mathbf{q}(\mathbf{x}, t)=-k \boldsymbol{\nabla} T(\mathbf{x}, t)
$$

dans $\mathcal{D}$

- Conditions aux limites thermiques

$$
\left\{\begin{array}{l}
T(\mathbf{x}, t)=T^{d}(\mathbf{x}, t) \\
\mathbf{q}(\mathbf{x}, t) \cdot \mathbf{n}(\mathbf{x}, t)=q^{d}(\mathbf{x}, t)
\end{array}\right.
$$

sur $\partial \mathcal{D}_{T}$

sur $\partial \mathcal{D}_{q}$ 


\section{Algorithme TRC}

L'algorithme TRC est établi pour résoudre les problèmes transitoires. La résolution est incrémentale en temps : à chaque pas de temps $\Delta t$, la matière est translatée d'une distance équivalente à $V \Delta t$. On présente la résolution du problème mécanique.

\subsection{Schéma de discrétisation par différences finies}

Il s'agit de discrétiser la relation :

$$
\frac{\partial \mathbf{f}}{\partial t}(\mathbf{x}, t)-\nabla_{\mathbf{x}} \mathbf{f}(\mathbf{x}, t) \cdot \mathbf{V}(t)=\lambda \frac{\partial f}{\partial \boldsymbol{\sigma}}(x, t)
$$

La discrétisation temporelle est arbitraire et dépend de la nature du problème étudié. La discrétisation spatiale est naturellement dictée par le transport des grandeurs physiques le long de lignes de courant. Ainsi, si l'on suppose que la vitesse $-\mathbf{V}$ de la structure par rapport au repère $\mathcal{R}^{\prime}$ est selon l'abscisse $x$, la dérivée de $\mathbf{f}$ devient :

$$
\dot{\mathbf{f}}(x, t)=\frac{\partial \mathbf{f}}{\partial t}(x, t)-V(t) \frac{\partial \mathbf{f}}{\partial x}(x, t)
$$

L'équation est résolue numériquement par un schéma aux différences finies du premier ordre, explicite en temps et décentré à droite en $x$ :

$$
\dot{\mathbf{f}}(x, t)=\frac{\mathbf{f}(x, t+\Delta t)-\mathbf{f}(x, t)}{\Delta t}+V(t) \frac{\mathbf{f}(x, t)-\mathbf{f}(x+\Delta x, t)}{\Delta x}
$$

Pour la loi d'écoulement plastique, le choix implicite est retenu [NGU 77]. Ainsi, la propriété de normalité est toujours respectée et le schéma est inconditionnellement stable. Finalement, la formulation discrétisée de la loi d'écoulement plastique est :

$$
\frac{\boldsymbol{\epsilon}^{\boldsymbol{p}}(x, t+\Delta t)-\boldsymbol{\epsilon}^{\boldsymbol{p}}(x, t)}{\Delta t}+V(t) \frac{\boldsymbol{\epsilon}^{\boldsymbol{p}}(x, t)-\boldsymbol{\epsilon}^{\boldsymbol{p}}(x+\Delta x, t)}{\Delta x}=\lambda \frac{\partial f}{\partial \boldsymbol{\sigma}}(x, t+\Delta t)
$$

\subsection{Résolution du problème discrétisé}

On note $(\mathrm{n}+1)$ le point à l'abscisse $x$, (n) le point à l'abscisse $x+\Delta x$, où $\Delta x$ est arbitraire (Figure 2). L'instant $t$ représente le temps initial. Chaque grandeur $\mathbf{f}$ est indicée par 0 quand elle est prise à la date $t . \Delta \mathbf{f}$ désigne l'incrément de $\mathbf{f}$ entre $t$ et $t+\Delta t$. La valeur recherchée est $\mathbf{f}(n+1, t+\Delta t)$.

Pour des raisons de minimisation du temps de calcul, le problème est supposé faiblement couplé. Les termes d'inertie et les dissipations plastiques sont négligés. La température est connue pour tous les instants. L'état mécanique de la structure est connu à l'instant $t$. Les contraintes $\sigma$ et les déformations plastiques $\epsilon^{p}$ sont à 


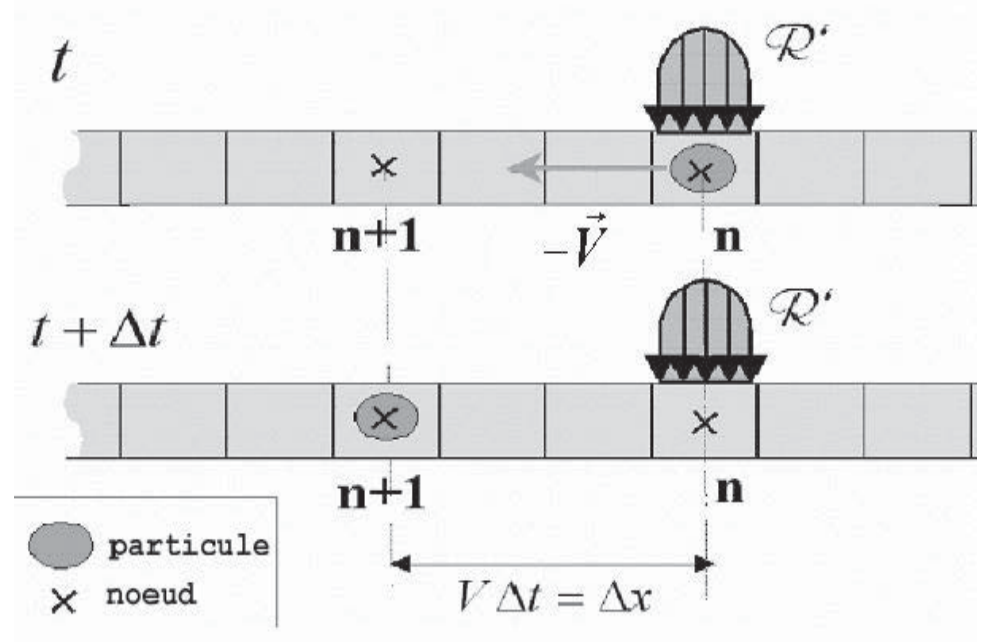

Figure 2. Schéma de l'algorithme TRC dans le cas où $\Delta x=V \Delta t$

déterminer pour le point $(n+1)$ à la date $t+\Delta t$ en fonction de $T_{o}, \boldsymbol{\sigma}_{o}, \boldsymbol{\epsilon}_{o}^{p}, \boldsymbol{\epsilon}_{o}^{p}(n)$ et $\Delta \epsilon$.

La résolution du système discrétisé donne :

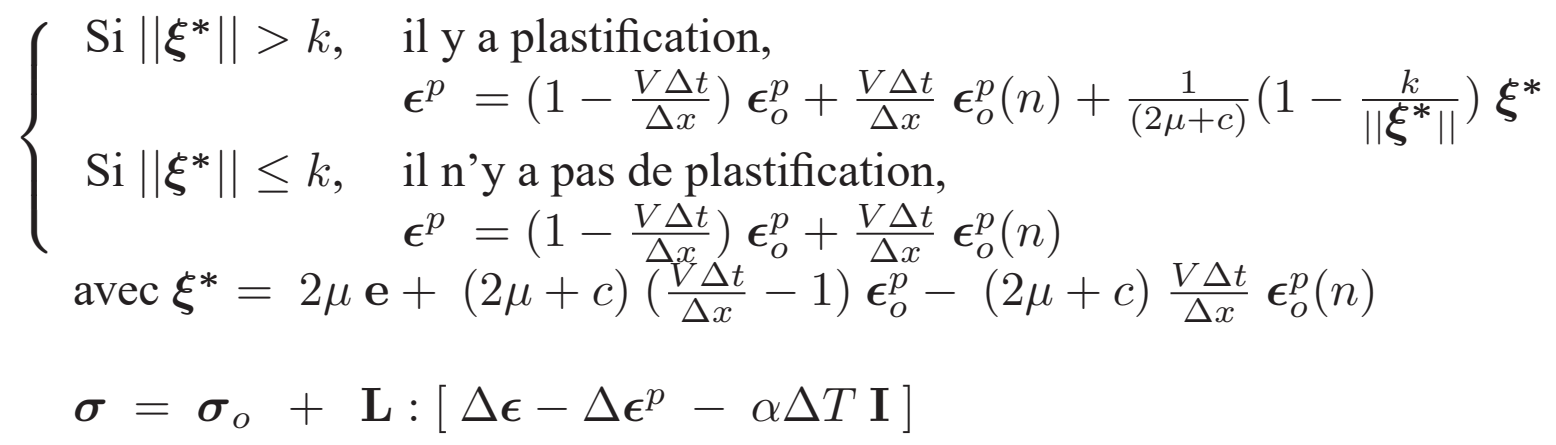

S'il n'y a pas de plastification,

$\frac{\partial \Delta \boldsymbol{\sigma}}{\partial \Delta \boldsymbol{\epsilon}}=\mathbf{L}$

S'il y a plastification,

$\frac{\partial \Delta \boldsymbol{\sigma}}{\partial \Delta \boldsymbol{\epsilon}}=\mathbf{L}-\frac{4 \mu^{2}}{2 \mu+c}\left(\mathbf{I}-\frac{1}{3} \mathbf{I} * \mathbf{I}\right)\left[\frac{\boldsymbol{\xi}^{*}{ }_{*} \boldsymbol{\xi}^{*}}{\left\|\boldsymbol{\xi}^{*}\right\|^{2}}+\left(\left\|\boldsymbol{\xi}^{*}\right\|-k\right)\left(\frac{1}{\left\|\boldsymbol{\xi}^{*}\right\|}-\frac{\boldsymbol{\xi}^{*}{ }^{*} \boldsymbol{\xi}^{*}}{\left\|\boldsymbol{\xi}^{*}\right\|^{3}}\right)\right]$

REMARQUE. - La formulation est non locale car les valeurs du point $(n+1)$ dépendent de celles du point $(n)$. Cette dépendance induit des programmations numériques non conventionnelles.

\subsection{Algorithme TRC}

Finalement, l'algorithme TRC en élastoplasticité ne diffère d'un algorithme incrémental classique que par la détermination des déformations plastiques. Ainsi, le problème peut être résolu avec l'algorithme de retour radial proposé par Nguyen Q.S. [NGU 77]. On remarque que si les termes d'inertie sont pris en compte, seule la rigidité du système est modifiée; le schéma de résolution reste inchangé. Le schéma de 
l'algorithme est donné dans le tableau 1. Les indices $j$ et $j+1$ désignent les numéros des itérations d'équilibre.

0 . Données à l'instant $t:\left\{\boldsymbol{\sigma}_{o}, \boldsymbol{\epsilon}_{o}, \boldsymbol{\epsilon}_{o}^{p}, T_{o}\right\}$

Données à l'instant $t+\Delta t:\{\Delta T\}$

Initialisation : $\left\{\Delta \boldsymbol{\sigma}_{j}, \Delta \boldsymbol{\epsilon}_{j}, \Delta \boldsymbol{\epsilon}_{j}^{p}\right\}=\{\mathbf{0}, \mathbf{0}, \mathbf{0}\}$

1. Données à l'instant $t_{j}=t+\Delta t_{j}:\left\{\Delta \boldsymbol{\sigma}_{j}, \Delta \boldsymbol{\epsilon}_{j}, \Delta \boldsymbol{\epsilon}_{j}^{p}\right\}$

2. Calcul de l'équilibre global et détermination de $\Delta \epsilon_{j+1}$

3. Test de plasticité

$$
\begin{aligned}
& \boldsymbol{\xi}_{j+1}^{*}=\boldsymbol{s}_{o}-H \boldsymbol{\epsilon}_{o}^{p}+2 \mu \Delta \boldsymbol{e}_{j+1}+(2 \mu+H) \frac{V \Delta t}{\Delta x}\left(\boldsymbol{\epsilon}_{o}^{p}-\boldsymbol{\epsilon}_{o}^{p}(n)\right) \\
& \text { Si }\left\|\boldsymbol{\xi}_{j+1}^{*}\right\| \leq k, \\
& \quad \text { incrément élastique : } \boldsymbol{\epsilon}_{j+1}^{p}=\boldsymbol{\epsilon}_{o}^{p}-\frac{V \Delta t}{\Delta x}\left(\boldsymbol{\epsilon}_{o}^{p}-\boldsymbol{\epsilon}_{o}^{p}(n)\right) \\
& \text { Sinon, } \quad \text { incrément plastique }: \boldsymbol{\epsilon}_{j+1}^{p}=\boldsymbol{\epsilon}_{o}^{p}-\frac{V \Delta t}{\Delta x}\left(\boldsymbol{\epsilon}_{o}^{p}-\boldsymbol{\epsilon}_{o}^{p}(n)\right)+\frac{1}{2 \mu+H}\left(1-\frac{k}{\left\|\boldsymbol{\xi}^{*}\right\|}\right) \boldsymbol{\xi}^{*} \\
& \text { Fin } \quad \\
& \boldsymbol{\sigma}_{j+1}=\boldsymbol{\sigma}_{o}+\mathbf{L}:\left[\left(\Delta \boldsymbol{\epsilon}_{j+1}-\Delta \boldsymbol{\epsilon}_{j+1}^{p}\right)-\alpha \Delta T \mathbf{I}\right]
\end{aligned}
$$

5. Test de convergence

Si convergence, aller à (0)

Sinon, $\quad$ aller à (1)

Tableau 1. Algorithme T.R.C. en élastoplasticité avec écrouissage cinématique linéaire

\section{Algorithme stationnaire passage par passage}

L'algorithme stationnaire passage par passage est développé pour les problèmes qui sont stationnaires pendant chaque passage du chargement. La méthode repose sur l'idée que, du point de vue du chargement, le problème est permanent au cours de chaque passage. On suppose également que la structure atteint un état résiduel indépendant de $\mathbf{x}$ une fois que le chargement est suffisamment loin.

L'hypothèse de régime permanent dans le repère du chargement se traduit par le fait que toutes les dérivées temporelles partielles sont nulles. Ainsi : 


$$
\dot{\mathbf{f}}(\mathbf{x})=-V \frac{\partial \mathbf{f}}{\partial \mathbf{x}}(\mathbf{x})
$$

Le schéma décentré à droite est adopté. La loi d'écoulement plastique devient :

$$
V \frac{\boldsymbol{\epsilon}^{p}(x)-\boldsymbol{\epsilon}^{p}(x+\Delta x)}{\Delta x}=\lambda \frac{\partial f}{\partial \boldsymbol{\sigma}}(x)
$$

On note $(n+1)$ le point se trouvant à l'abscisse $x ;(n)$ est le point à l'abscisse $x+\Delta x$ comme indiqué sur la figure 3 . On cherche les valeurs au point $(n+1)$.

Le problème est résolu passage par passage. L'état initial connu de la structure est constant par ligne de courant. L'état thermomécanique du point $(n)$ est établi pour la date $t_{0}+\Delta t$. Les contraintes $\boldsymbol{\sigma}$ et les déformations plastiques $\boldsymbol{\epsilon}^{p}$ doivent être déterminés pour le point $(n+1)$ à la date $t_{0}+\Delta t$ en fonction de $T_{o}, \boldsymbol{\sigma}_{o}, \boldsymbol{\epsilon}_{o}^{p}$ et $\Delta \boldsymbol{\epsilon}^{p}(n)$.

Les expressions des déformations plastiques sont alors :

$$
\begin{aligned}
& \begin{cases}\text { si }\left\|\boldsymbol{\xi}^{*}\right\|>k, & \text { il y a plastification, } \\
& \boldsymbol{\epsilon}^{p}=\boldsymbol{\epsilon}_{o}^{p}+\Delta \boldsymbol{\epsilon}^{p}(n)+\frac{1}{(2 \mu+c)}\left(1-\frac{k}{\left\|\boldsymbol{\xi}^{*}\right\|}\right) \boldsymbol{\xi}^{*} \\
\text { si }\left\|\boldsymbol{\xi}^{*}\right\| \leq k, & \text { il n'y a pas de plastification, } \\
& \boldsymbol{\epsilon}^{p}=\boldsymbol{\epsilon}_{o}^{p}+\Delta \boldsymbol{\epsilon}^{p}(n)\end{cases} \\
& \operatorname{avec} \boldsymbol{\xi}^{*}=\mathbf{s}_{o}-c \boldsymbol{\epsilon}_{o}^{p}+2 \mu \Delta \mathbf{e}-(2 \mu+c) \Delta \boldsymbol{\epsilon}^{p}(n)
\end{aligned}
$$

Les expressions des contraintes et des matrices tangentes sont les mêmes que celles établies pour l'algorithme TRC.

REMARQUe. - S'il y a de la plastification, les déformations plastiques en $(n+1)$ s'écrivent: $\boldsymbol{\epsilon}^{p}=\boldsymbol{\epsilon}_{o}^{p}+\Delta \boldsymbol{\epsilon}^{p}(n)+\frac{1}{(2 \mu+c)}\left(1-\frac{k}{\left\|\boldsymbol{\xi}^{*}\right\|}\right) \boldsymbol{\xi}^{*} . \Delta \boldsymbol{\epsilon}^{p}(n)$ correspond au terme convectif qui rend compte du transport des quantités le long des lignes de courant, tandis que $\frac{1}{(2 \mu+c)}\left(1-\frac{k}{\left\|\boldsymbol{\xi}^{*}\right\|}\right) \boldsymbol{\xi}^{*}$ donne l'incrément temporel de déformations plastiques. Il apparaît clairement que $(n)$ doit être calculé avant $(n+1)$. En supposant que les valeurs initiales des points (0) sont connues, l'état des points (1) peuvent être déduits. De point en point, l'état thermomécanique de toute la structure peut être déterminée. Les points de référence $(m)$ illustrent l'état résiduel de la structure une fois que le chargement a effectué un passage : ils donnent l'état initial de la structure pour le passage suivant (une valeur par ligne de courant). $(m)$ est pris loin des frontières de la structure pour éviter tout effet de bord.

En conclusion, cette méthode de résolution permet de déterminer la réponse thermomécanique de la structure après chaque passage du chargement, en un seul calcul. Les lois ne sont pas locales et les calculs doivent être effectués dans un ordre lié aux lignes de courant. 


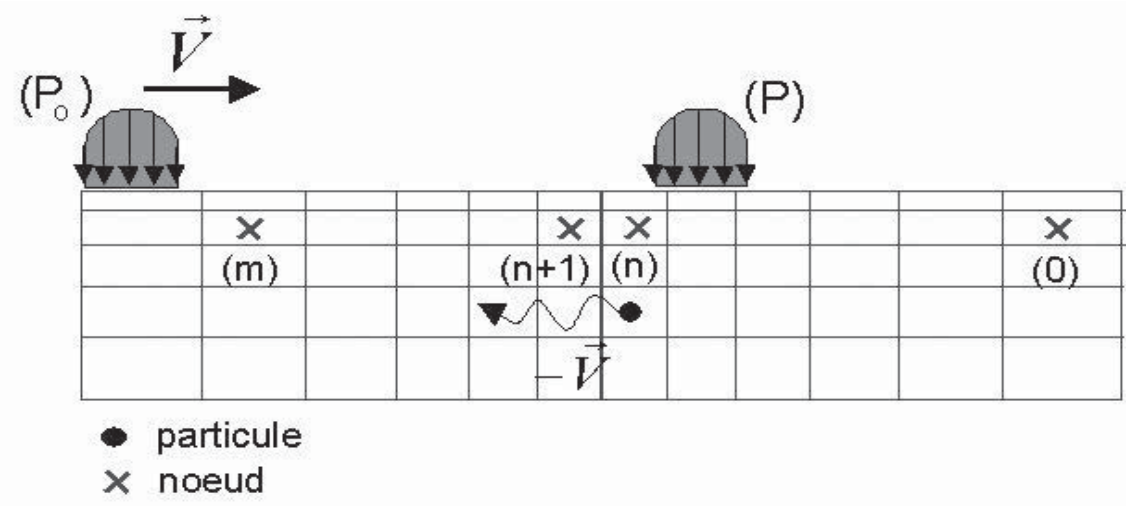

Figure 3. Schéma de l'algorithme stationnaire passage par passage

\section{Illustration sur une structure 2D}

L'algorithme TRC (ainsi que l'algorithme stationnaire) a été implémenté dans le logiciel Abaqus, à l'aide des user subroutines UMATHT et UMAT. Ces routines permettent à l'utilisateur du logiciel de programmer le comportement thermique et mécanique du matériau étudié. Afin de valider l'algorithme, un calcul en élastoplasticité à écrouissage cinématique linéaire a été effectué sur une structure bidimensionnelle rectangulaire. La vitesse de translation de la structure est $V=-1 \mathrm{~ms}^{-1}$. On impose un chargement d'amplitude $F=500 M N$. Les conditions aux limites sont données sur la figure 4. Les coefficients mécaniques du matériau sont présentés dans le tableau suivant :

\begin{tabular}{|l|c|}
\hline module de Young & $E=210 \mathrm{GPa}$ \\
\hline coefficient de poisson & $\nu=0.27$ \\
\hline limite d'élasticité en traction & $\sigma_{y}=340 \mathrm{MPa}$ \\
\hline coefficient d'écrouissage & $c=12 \mathrm{GPa}$ \\
\hline
\end{tabular}

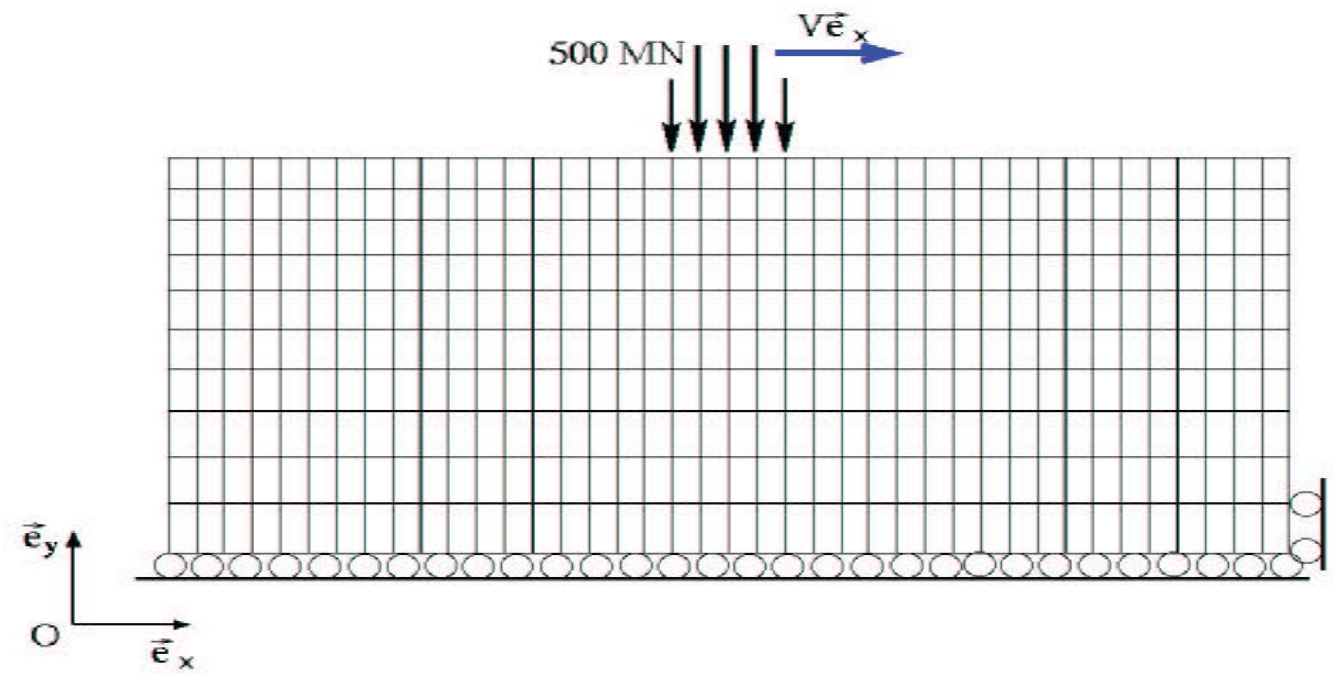

Figure 4. Exemple d'une structure rectangulaire 2D 
Trois maillages ont été utilisés :

- un maillage "grossier" à 400 éléments (à 4 points de gauss) qui permet de valider l'algorithme TRC (Figure 4);

- un maillage irrégulier à 540 éléments qui met en évidence les avantages de la méthode TRC (Figure 6, Figure 8);

- un maillage fin à 800 éléments qui sert de référence (Figure 7).

On compare les résultats issus d'un calcul "incrémental classique" (qui utilise les options *ELASTIC et *PLASTIC d'Abaqus), dans lequel le chargement est translaté à chaque pas de temps d'une distance équivalente à la longueur d'un côté d'élément, et d'un calcul résolu avec l'algorithme TRC, où $V \Delta t=\Delta x$ (Figure 5). Les résultats montrent une parfaite adéquation entre les deux solutions sur les premiers incréments de temps, ce qui valide l'algorithme TRC. Les différences que l'on perçoit à partir de l'instant $t_{0}+12 \Delta t$ sont dues au fait que dans le calcul "classique", le chargement finit par subir des effets de bord. La méthode TRC permet donc une meilleure évaluation des structures très longues pour lesquelles les effets de bord ne doivent pas intervenir sur la solution sous le chargement (problème du rail, du laminage...).

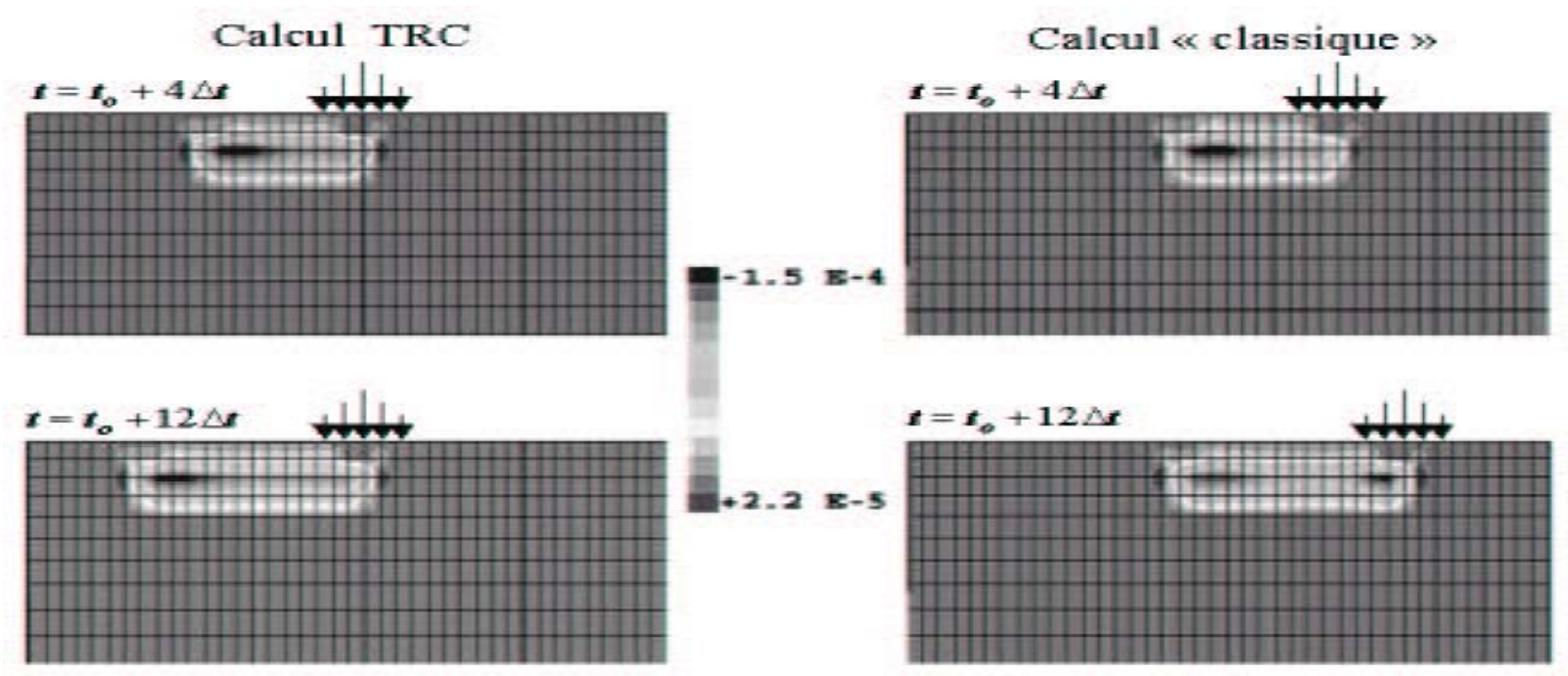

Figure 5. Comparaison de calculs : déformations plastiques selon $\mathbf{e}_{\mathbf{y}}$

Pour mettre en avant les avantages des deux algorithmes TRC et stationnaire, quatre calculs différents ont été effectués :

- un calcul TRC avec le maillage irrégulier;

- le même calcul avec le maillage fin, qui sert de référence;

- le même calcul résolu avec l'algorithme stationnaire passage par passage;

- un calcul dont l'amplitude du chargement varie cycliquement sur un passage de $F=200 M N$ à $F=500 M N$.

Les isovaleurs des déformations plastiques dans la direction $\mathbf{e}_{\mathbf{y}}$ sont données dans les figures $6,7,8$ et 9 . 


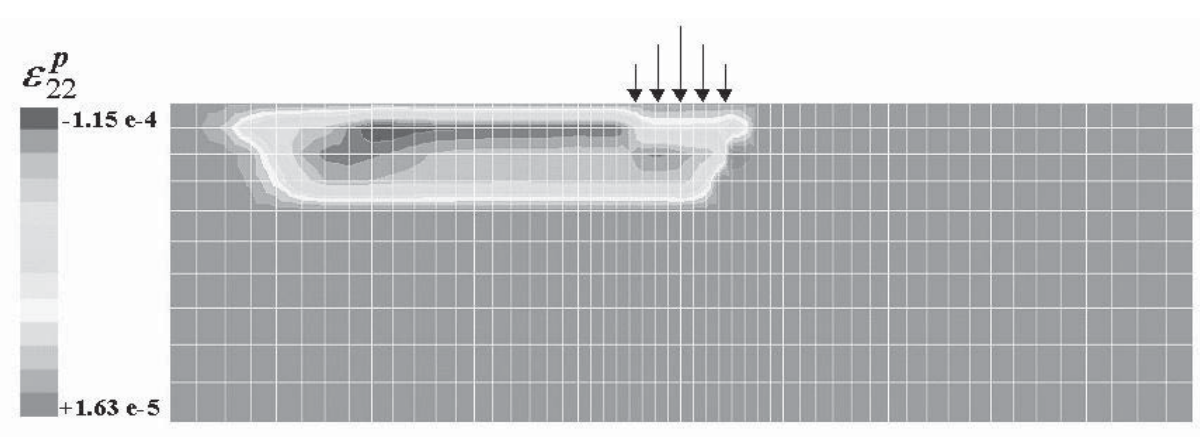

Figure 6. Calcul TRC avec un maillage irrégulier

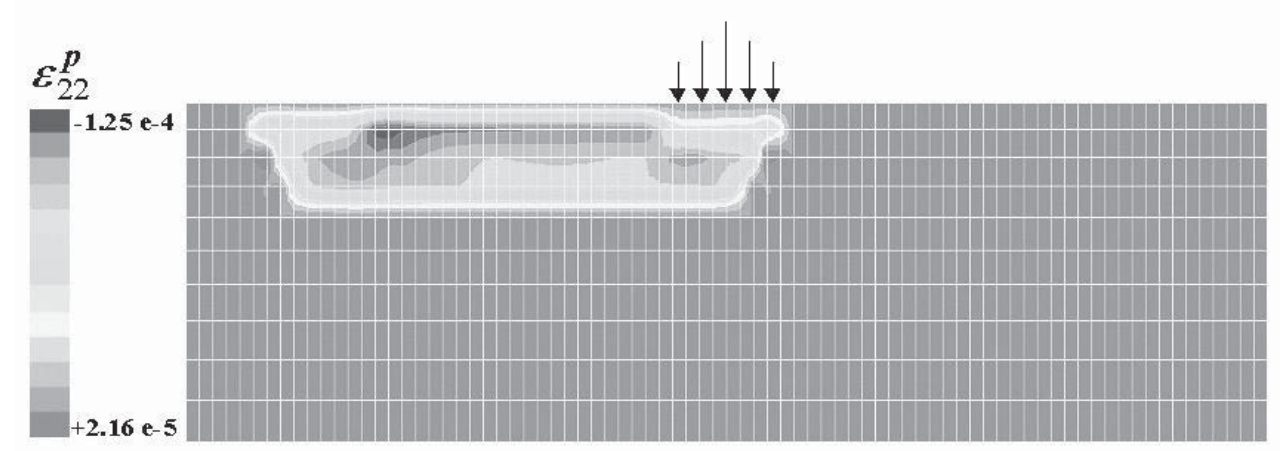

Figure 7. Calcul TRC avec un maillage fin

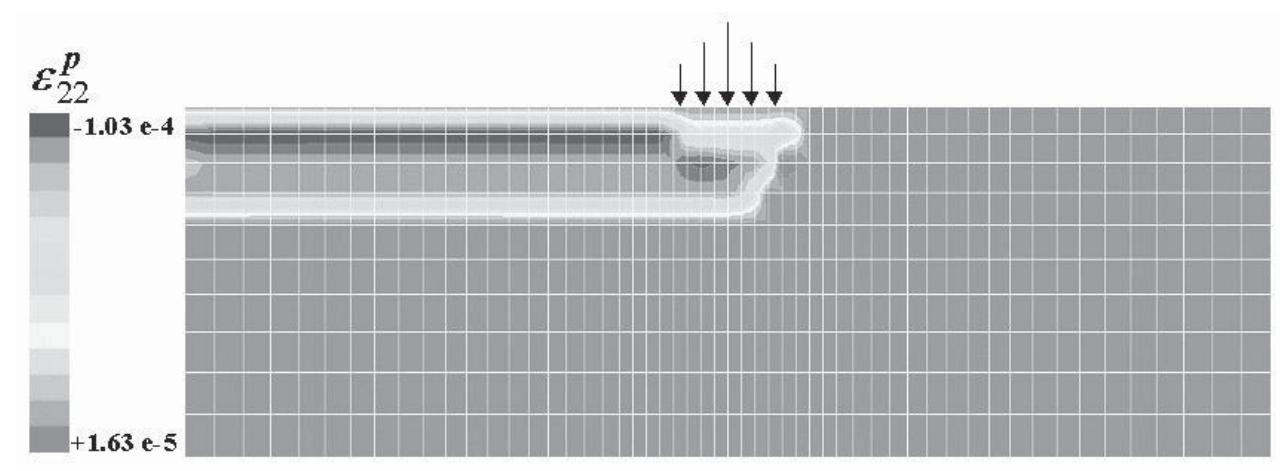

Figure 8. Calcul stationnaire pour un passage de chargement

Les calculs TRC montrent que le maillage irrégulier permet une bonne évaluation des gradients sous le chargement. Une petite différence peut être observée loin de cette zone mais l'économie de temps de calcul reste intéressante. En effet, le calcul transitoire avec le maillage fin prend $201 \mathrm{~s}$ CPU, tandis que le calcul avec le maillage irrégulier ne dure que $133 \mathrm{~s}$ CPU. Il est clair que le gain de temps de calcul sera encore plus notable dans des analyses de structures tridimensionnelles. Le calcul stationnaire est très court : $25 \mathrm{~s}$ CPU. La valeur stabilisée obtenue $\left(\epsilon_{y y}^{p}=1.0310^{-4}\right)$ est très proche de celles atteintes avec les calculs TRC $\left(\epsilon_{y y}^{p}=1.0410^{-4}\right.$ avec le maillage irrégulier et $\epsilon_{y y}^{p}=1.0210^{-4}$ avec le maillage fin). L'efficacité de cette méthode stationnaire justifie amplement son utilisation dès lors que les hypothèses de l'algorithme sont respectées. L'algorithme TRC doit, quant à lui, être utilisé à chaque fois que le 
chargement varie au cours d'un passage. Le dernier calcul montre la réponse cyclique de la structure à un chargement lui-même cyclique.

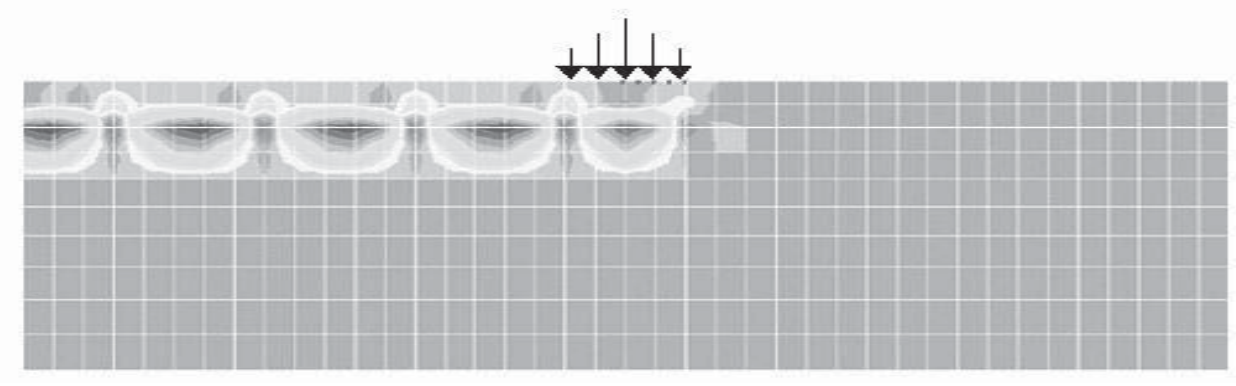

Figure 9. Calcul TRC avec un chargement d'amplitude variable

Cet exemple permet de conclure sur les avantages des algorithmes développés :

- les domaines où les chargements et les conditions aux limites sont imposés sont fixes. La mise en œuvre de l'analyse est donc simple;

- pour des structures très longues, les effets de bords peuvent être ignorés ;

- le maillage n'est fin que sous le chargement; les rendements des calculs sont meilleurs en termes de précision/temps de calcul;

- les variations de l'amplitude et de la vitesse du chargement peuvent être simulés (au cours d'un passage avec l'algorithme TRC et entre deux passages avec l'algorithme stationnaire).

\section{Simulation numérique d'un disque de frein}

La simulation numérique d'un disque de frein automobile est présentée dans cette partie. Le système de freinage est principalement composé d'un disque en fonte fixé à la roue par l'intermédiaire d'un moyeu, de deux plaquettes de friction et d'un étrier qui les maintient. Les plaquettes et l'étrier sont solidaires de la carosserie. Lorsque le véhicule est en mouvement, le disque est en rotation. Pendant la phase de freinage, les deux plaquettes sont pressées contre le disque, de sorte que son mouvement est ralenti. Le système s'apparente donc bien à un problème de structure (le disque) soumise à des chargements mobiles (pression des plaquettes et chaleur induite par frottement).

Au cours d'un freinage, l'énergie cinétique du véhicule (de l'ordre de 300-600 kJ) est essentiellement transformée en énergie thermique à l'interface entre les plaquettes et le disque. Le flux thermique reçu par le disque au niveau du contact disque/plaquettes engendre alors de fortes élévations de température (la température moyenne du disque est proche de $300^{\circ} \mathrm{C}$ ) et de très grands gradients thermiques (la température peut atteindre localement $700^{\circ} \mathrm{C}$ ). Ces sollicitations thermiques endommagent le disque sous forme de faïençage sur les pistes de frottement. Dans des cas d'utilisation très sévère sur banc d'essai, le disque peut également fissurer radialement ou même rompre au niveau du bol. Afin de prédire ces comportements, il faut évaluer avec précision l'histoire thermique du disque, ainsi que sa réponse mécanique. 
L'existence des gradients thermiques (dans l'épaisseur du disque mais aussi dans le sens orthoradial) impose que le maillage soit tridimentionnel non axisymétrique et très fin dans l'épaisseur des pistes de frottement et sous les plaquettes. Les durées de freinage (environ 4-10s) ne permettent pas d'atteindre une réponse thermique stationnaire. Le problème est transitoire. L'algorithme TRC est donc utilisé pour la simulation thermique du freinage. Etant données les gammes de température de fonctionnement du disque, le comportement mécanique de la fonte ne peut pas être considéré comme purement élastique. La simulation doit prendre en compte la plasticité, ce qui impose un calcul itératif long. L'hypothèse de régime permanent au cours de chaque tour de disque est pertinente. Le problème mécanique est donc résolu avec l'algorithme stationnaire passage par passage. Il y a autant de calculs élastoplastiques que de tours de disque.

Le maillage $3 \mathrm{D}$ contient 4050 hexaèdres à 8 points de gauss. Le freinage simulé dure $\Delta t=4 \mathrm{~s}$. La vitesse angulaire du disque décroît linéairement de $\omega_{0}=$ $105 \mathrm{rads}^{-1}$ (correspondant à une vitesse de véhicule de $100 \mathrm{~km} / \mathrm{h}$ ) à $\omega=0 \mathrm{rads}{ }^{-1}$. Le chargement thermique est un flux de chaleur entrant localisé sous les plaquettes. Il est calculé à partir de la perte d'énergie cinétique du véhicule. Durant le calcul mécanique, le disque subit une pression uniforme égale à 80 bars, localisée sous les plaquettes.

Les cartes de température du disque en début et fin de freinage, ainsi que les contraintes de von Mises après refroidissement sont données dans les figures 10 et 11. On note la non-axisymétrie de la carte de température. La valeur maximale obtenue $\left(T=279^{\circ} \mathrm{C}\right)$ est très proche de celle mesurée par un thermocouple frottant $\left(T=265^{\circ} C\right)$. Le maximum des contraintes est bien obtenu au niveau du bol, où la rupture peut démarrer. Les temps de calcul sont tout à fait satisfaisants puisqu'il faut $13100 s$ CPU pour simuler le freinage sur un HP780/180. Cet outil de calcul permet aujourd'hui d'aborder différentes simulations de freinage. Des corrélations avec des essais sur un banc dynamométrique instrumenté en thermocouples, pyromètre et rosettes sont en cours de réalisation, avec des améliorations de maillage, de conditions aux limites, de modèles rhéologiques, etc. [NGU 02].

\section{Conclusion}

Une méthode de calcul eulérien qui permet de traiter les problèmes de structures subissant des chargements mécaniques et/ou thermiques mobiles a été présentée. Sa formulation dans l'hypothèse des petites transformations et son schéma de résolution ont été donnés, pour les cas transitoires et les cas stationnaires. L'implémentation de ces algorithmes TRC et stationnaire dans le code de calcul Abaqus permet d'aborder des problèmes industriels complexes comme celui du disque de frein automobile. Cet exemple montre l'intérêt des algorithmes en termes de temps de calcul. 

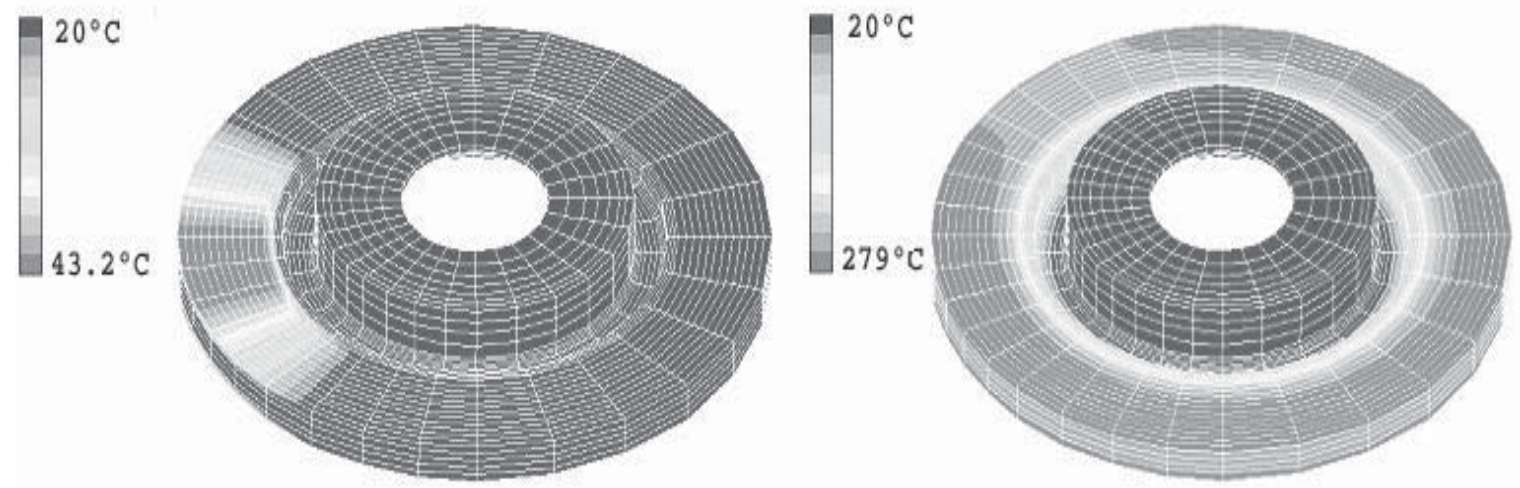

Figure 10. Température en début et fin de freinage
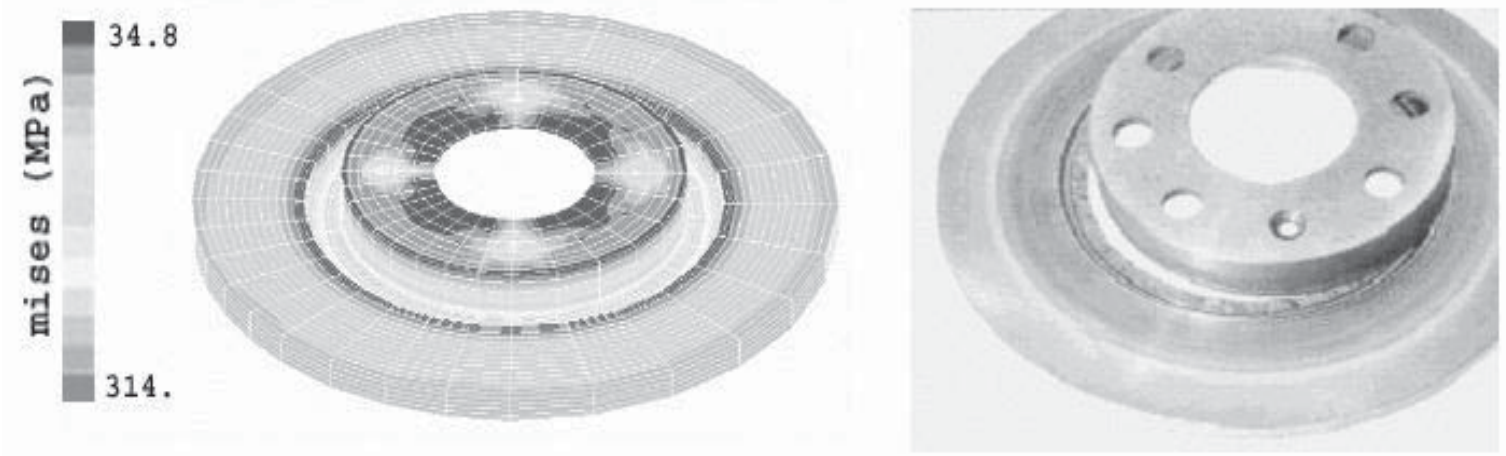

Figure 11. Contraintes de von Mises après refroidissement

\section{Bibliographie}

[ASK 97] Askes H. , "Mesh adaptivity methods : the ALE technique for localisation", TU Delft - Faculty of Civil Engineering, Mechanics \& Structures Computational Mechanics, march 1997.

[DAN 93] Dang VAn K., Maitournam M.H. , "Steady -State Flow in classical elastoplasticity : Applications to repeated rolling and sliding contact", J. Mech. Phys. Solids, Vol.41, $n^{o} 11$, p. 1691-1710, 1993.

[NGU 77] NGUYEN Q.S. , "On the elastic plastic initial-value problem and its numerical integration", Int. J. Num. Meth. Engng. 11, p. 817-832, 1977.

[NGU 81] NGUyen Q.S., Rahimian, "Mouvement permanent d'une fissure en milieu élastoplastique", J. de Mech. Appl., 5, p. 95-120, 1981.

[NGU 02] NGUyen-TAjan T.M.L. , "Modélisation thermomécanique du disque de frein par une approche eulérienne", Thèse de doctorat, Ecole Polytechnique, 2002.

[ZAR 80] ZARKA J., , "Direct analysis of elastic-plastic structures with overlay materials during cyclic loading", Int. J. Num. Meth. Engng 15, p. 225-235, 1980. 